\title{
The Research and Application Suggestions of Degradable Macromolecule Materials
}

\author{
Xiaotian Wang, Jinyu Wei \\ School of management, Tianjin University of Technology, Tianjin 300384, China
}

Keywords: biodegradation, degradable macromolecule, macromolecule materials.

\begin{abstract}
In this paper, reviewed some biodegradable polymer materials such as photodegradable polymeric materials, chemical synthesis polymer materials, microbial synthesis polymer materials, photo biodegradable polymeric materials and mixed type polymer materials. Points out the existing problems and the development directions of degradable polymeric materials. And some suggestions for future development.
\end{abstract}

\section{Introduction}

The widespread application of polymer material products caused a lot of waste, which has become the concern of the international community and serious environmental pollution problems to be solved. Using the traditional incineration, burial and other processing techniques have some deficiencies, and recycling often has certain limitation, so developing the degradable polymer material which can be accepted by environment is the way to solve waste disposal problem of polymer materials, especially the important way for the pollution problems of goods which is hard to recycle disposable, which has become a hot topic at the field of polymer [8]. Degradable macromolecule materials waste in the natural environment, with the effect of various environmental factors for a certain time can automatically decomposed into small molecular substances with no environmental pollution, and which even can be involved in metabolism cycle and assimilation.

Biodegradable macromolecule materials refers to the polymer materials after used in the specific environmental conditions, with the effect of some environmental factors such as light, wind, water, oxygen, microorganisms, insects and mechanical force[9], whose the chemical structure can change significantly in a short period of time, thus causing biological deterioration, which eventually is absorbed by environment. According to the different dissolution mechanism, degradable macromolecule materials can be divided into photodegradable polymeric materials, biodegradable polymer materials, photo biodegradable polymer materials, oxidative degradation of polymer materials, composite degradable polymeric materials and so on, wherein the biodegradable polymer materials refers to the polymer materials under the effect of tissue cells, enzymes and body fluid in natural microorganisms or in human and animal body, make its chemical structure changes, which cause the change in molecular weight decreases and the performance[1]. Application of biodegradable polymeric materials is widely, and which has broad application prospects in the packaging, food and catering industry, disposable daily necessities, drug controlled release system, clinical medicine, medical equipment and other fields, so the development of biodegradable polymer materials have become the focus of research in the world.

\section{Photodegradable polymeric materials}

Polymer degrade into low molecular weight compound which is environmental safety by absorbing optical energy under the effects of optical-oxygen in the light (mainly the ultraviolet light), which caused chain scission reaction induced by light and free radical oxidative chain scission reaction that is Norrish photochemical reaction. This kind of light sensitive polymer material is called photodegradable polymeric materials, according to the preparation method can be divided into two types such as synthetic type and addition type. 
Synthetic type. Synthesis photodegradable polymeric materials is endowed with the light degradation characteristics which is mainly through the copolymerization reaction in the polymer main chain by introducing carbonyl type photosensitive groups, and which can control the light degradation activity by regulating the carbonyl group content[4, 10]. Usually using photosensitive monomer $\mathrm{CO}$ or ketene (such as methyl vinyl ketone, methyl acrylic ketone) copolymerization with olefin monomers, can synthesize photo degradation type PE, PP, PS, PVC, PET and PA which contain carbonyl structure, most is the study of photo degradation polymers of vinyl copolymert, this is because the oligomers which molecular weight is less than 500 from the degradation of PE can be absorbed and degraded by the soil microbial, which is safe to environment. At present, the photo catalytic degradation of polymers that has been the realization of industrialization is ethylene-CO copolymer and ethylene-vinyl ketone copolymer, which can be used for plastic film, packing bag, container, fiber, sheet, foam products etc.

Addition type. The photodegradable polymeric materials can be made by adding photosensitive agent to the common polymer material, under the effect of light, the photosensitizer can be dissociated into free radicals with activity, and then cause the broken chain reaction of polymer molecular which achieved degradation. General photosensitizers are: transition metal complexes (such as two thiocarbamate), stearic acid salt (such as iron stearate), halide (such as N-halogenated hydantoin), carbonyl compounds (such as anthraquinone, ketone compounds (such as two benzophenone), polynuclear aromatic compounds (such as Mao) and some photosensitive polymers (such as poly isobutylene) and synthetic light degradable polymer etc. Photo degradation characteristics depend on the species composition and quantity of photosensitizer, etc.

\section{Chemical synthesis polymer materials}

Due to ester is easy to be decomposed by microorganisms or enzymes in nature, so chemical synthesis of biodegradable polymer materials are mostly aliphatic polyester of ester structure contained in the molecular structure. Polyester and polyester copolymer can be obtained by diols and diacids (or dicarboxylic acid derivatives), stepwise polymerization of hydroxy acid, which can also be prepared by ring opening polymerization of lactone ring. The influence of polycondensation reaction is due to the degree of reaction and the water or other small molecules in the reaction process, it is difficult to get high molecular weight products. Ring opening polymerization is only affected by the activity of the catalyst and the outside conditions, can obtain high molecular weight polyester, which molecular weight is up to 106 , and the monomer is completely conversion polymerization. Aliphatic polyester has the shortcomings of low melting point, poor mechanical properties and the high hydrophobicity, so the study of modification is active. Therefore, ring opening polymerization become an ideal polymerization method way of homopolymerization and copolymerization synthetic biodegradable polymer material of lactone $[3,5]$, glycolide and lactide. The current main products that have been developed are polylactic acid (PLA), polycaprolactone (PCL), polybutylene glycol butyl ester (PBS two) etc. Table 1 compares the performance of various copolymers. In addition to aliphatic polyester, polyphenols, polyaniline, polycarbonate, poly aspartic acid have also been successfully developed.

Table 1 PBS and its properties of copolyester

\begin{tabular}{c|c|c|c|c|c|c|c}
\hline Polymer & $\begin{array}{c}\text { The feeding } \\
\text { molar ratio }\end{array}$ & $\begin{array}{c}\text { Reaction } \\
\text { time/h }\end{array}$ & $\mathrm{Mn} \times 10^{-4}$ & $\mathrm{Mw} / \mathrm{Mn}$ & $\mathrm{Tm} /{ }^{\circ} \mathrm{C}$ & $\mathrm{Tg} /{ }^{\circ} \mathrm{C}$ & $\begin{array}{c}\mathrm{Td}(-2 \%) / \\
{ }^{\circ} \mathrm{C}\end{array}$ \\
\hline PBS & $100: 0$ & 3 & 8.63 & 1.97 & 114 & -35.7 & 348 \\
P(BS-co-CL) & $96: 4$ & 4 & 8.80 & 2.0 & 112 & -33 & 312 \\
P(BS-co-LA) & $95: 5$ & 2 & 6.99 & 2.0 & 110 & -30 & 313 \\
P(BS-co-DGA) & $95: 5$ & 1.3 & 8.01 & 2.19 & 111 & -34.6 & 318 \\
P(BS-co-CHDH) & $95: 5$ & 1.7 & 10.46 & 2.08 & 107 & -30.5 & 313 \\
\hline
\end{tabular}

Synthetic polymer material has more advantages than the natural macromolecule material, which can design the structure of molecular chain from the angle of molecular chemistry, so as to control the 
physical properties of polymer materials, but also can make full use of a variety of small molecule monomers which are synthetic or extracted from natural. But in how to precisely control the performance by designing the molecular structure have yet to be further study.

\section{Natural polymer materials}

Natural polymer material such as starch, cellulose, hemicellulose, lignin, pectin, chitin, protein rich source, low price, especially the natural production ranks first in the biosynthesis of cellulose and chitin, the annual volume of more than $10^{10}$ tons. Use them for preparation of biopolymer materials can be degraded completely, has good biocompatibility, non-toxic, double meaning formed thereby both products of natural regeneration of making full use of resources and environmental governance, therefore attention all over the world, especially in Japan. Such as industrial technology experiment Japan with cellulose and chitin was prepared from chitosan composite, using film casting workmanship, with strength and general film the same, and can be completely degraded after 2 months; they also of chitosan - starch composite polymer materials conducted a lot of research work, found to regulate the proportion of raw materials, heat treatment temperature, can change the intensity and time of degradation of polymer materials.

Although natural polymer material has many advantages, such as low price of complete degradation, but its thermodynamic performance is poor, cannot meet the requirements of the performance of polymer materials processing engineering, blend between so by chemical modification of natural polymer, natural polymer and natural polymer and synthetic polymers blends to produce good degradability, biodegradable polymer material and practical sex is at present one of the main research direction.

\section{Present situation and development suggestion of macromolecule materials}

Photodegradable polymeric materials technology is relatively mature, which market share at present is up to $70 \% \sim 80 \%$, mainly used for plastic bags, partly used in agricultural film. But because of the limitation of the method of degradation, its application and development will face challenge in the future.

Biodegradable polymer materials is still in developing stage, which has high technology content and good application prospect. The main problems of chemical synthesis and microbial synthesis type at present is the product cost is too high, the application field is restricted, mainly used in some special fields such as medical materials etc.. Chemical synthetic type in the future should pay attention to improve product performance, innovate synthesis technology and use of inexpensive raw materials (such as $\mathrm{C} 1$ raw material methanol, CO, CO2); Microbial synthesis type should focus on the development of high efficient bacteria, use of inexpensive raw materials, the fermentation technology optimization and improvement of extraction technology and so on, try to reduce the cost of products and expand the application market. The transgenic biological production is an important direction of degradable polymer materials that cannot be neglected, which belongs to the front research, this challenging field has gradually attracted people's attention. Natural polymer type and mixed type of biodegradable polymer materials, there are many technical problems need to be further studied, including starch filled because there is now a problem of incomplete degradation is eliminated, so the domestic production of starch filled based biodegradable resin production enterprises status and prospects of poor. According to some people think except the application prospect in some special areas of biodegradable polymer materials of the bleak, especially natural polymer type and mixed type uncertainty. In fact, in addition to the copolymerization modification of various biodegradable polymer materials by blending modification preparation of biodegradable polymer material with good comprehensive properties will still be an important technical approach, especially the natural polymer and blend the corresponding fully biodegradable polymer materials due to its full utilization of cheap and abundant renewable natural resources ${ }^{[9]}$. It will be important to breed with good prospects for the application of biodegradable polymer materials, the success of "Novon" is a good 
example of. In addition, more attention should be paid for the study of controllable degradation and biodegradation of accelerators, clear professional term, making performance evaluation method and standard unified and effective [7].

\section{Conclusion}

Biodegradable polymer materials exists the potential market and has the very good prospects for development in the disposable daily necessities, fishing net, diapers, sanitary napkins, cosmetics, shoes, caps, gloves, sleeve tablecloth, gardening and other aspects except in application of packaging, catering industry, agriculture, medicine field. For the conclusion of degradation products and environmental safety problems should be cautious, and should also develop the research of comprehensive experimental application.

\section{References}

[1] AiJun He. Chemical model of polymer materials, Vol.30 (2002), p.1-7. (In Chinese)

[2] Chen Y, Chen J, Yu C. Proc Biochem, Vol.34 (1999), p.153-157.(In Chinese)

[3] GuoQiang Chen, Kai Zhao. Chinese Biotechnology, Vol.22 (2002), p.1-8. (In Chinese)

[4] Jun $\mathrm{Xu}$, WenNan He, BaoHua Guo.Institution of Higher Learning Chemical Journal, Vol.23 (2002), p.71-74. (In Chinese)

[5] Jing Qiu, Qiong Wu, Guang Zhang. Food and Biotechnology, Vol.21 (2002), p.76-79. (In Chinese)

[6] ShengQi Shen. Tianjin Chemical, Vol.2 (2002), p.28-29.(In Chinese)

[7] ShenGuo Wang. Chemical Bulletin, Vol.2 (1997), p, 45-48.(In Chinese)

[8] Shankar W R, Thachil E T.Polym Recycl, Vol.4 (1999), p.101 -115.

[9] Scott G .Polym Degrad Stab, Vol.68 (2000), p.1-7.

[10]Xianliang Song. Polymer Bulletin, Vol.4 (2002), p.47-52. (In Chinese)

[11]Zhao K, Deng Y, Chen J C. Biomaterials, Vol.24 (2003), p.1041-1045. (In Chinese) 